\title{
THE WINDING MECHANICS OF LAMINATE WEBS
}

\author{
By \\ Sheng Pan, Ron Markum and J. K. Good \\ Oklahoma State University \\ USA
}

\begin{abstract}
Models that describe the residual stresses due to winding single layer webs at the end of roll-to-roll manufacturing machines are mature. These models have been used to reduce or avoid defects that are due to winding. Many laminated products exist where two or more webs have been joined to form a thicker composite web. The properties of each layer provide a unique functionality to the product. No laminate winding models exist in the literature. This paper will focus on the development of a laminate winding model and lab test verification of the model.
\end{abstract}

\section{INTRODUCTION}

Roll-to-Roll (R2R) manufacturing processes constitute a large sector of all manufacturing. The materials used in these processes are very long, quite thin, and susceptible to damage. R2R manufacturing involves additive processing that is rate dependent. One or more base webs must be formed. The web may be coated uniformly or selectively with one or more coatings depending on product needs. In some cases the web will be laminated to other webs that may have their unique coatings. Finally the web is cut to shape and becomes a product or part of a product. The web formation, the coating(s), laminating, etc. all occur in unique process machines due to the different rates at which these processes can occur. This requires the web to be stored and historically the only available means has been to wind the webs into rolls.

Winding is often detrimental to web and product quality. Roll defects are inevitable in the winding process, such as roll telescoping, roll blocking, buckling, bulk loss etc., leading to inestimable economic loss. Many web defects are caused by residual stresses due to winding and prevention of the defects requires a means or model for determining the stresses. A winding model is a prediction of the wound roll residual stresses based on knowledge of winding tension, web and core geometric and material properties, and the finish radius of the roll to be wound. 
Laminating and coating are common R2R additive manufacturing processes. Often products require multiple layers that are joined by some method. Coating of web materials is common, the coating is often considered non-structural since the machine direction (MD) stiffness per unit length (the product of modulus and cross sectional area) is small compared to that of the web. The coating will likely alter the surface of the web and affect the contact mechanics between web layers in the roll. This will also likely affect the apparent radial modulus of elasticity of the wound roll and hence the winding residual stresses. Even so previously developed winding models have the capacity to predict the residual stresses in rolls wound from coated webs.

Laminates differ from coated webs in that the axial stiffness of the webs to be joined are comparable. Practiced use of existing models [1-4] have shown that the winding tension has more influence on the development of the winding residual stresses than any other input. Laminates require two or more webs to be laminated and are often subjected to unique tensions prior to lamination. These unique tensions may be chosen in an effort to match the MD strains in all layers prior to lamination. This practice is known as strain matching and when done the objective is usually to minimize MD curl. Laminated webs that are not strain matched will curl when cut into discrete products which is typically undesirable. In some cases strain matching is not possible as one or more layers may suffer transport defects such as wrinkling at the tension that would be required to match the MD strains of all layers. Any model that is developed should be robust and allow the user to select whether strain matched or non-strain matched conditions at the laminator.

\section{BACKGROUND}

Several one dimensional (1D) winding models have been developed for the winding of single layer webs. The one dimension is in the radial direction and these models would predict tangential $\left(\sigma_{\theta}\right)$ and radial $\left(\sigma_{\mathrm{r}}\right)$ stresses as a function of radial position within the roll. Those models that were most useful [1-4] were developed acknowledging the wound web material to be orthotropic with a radial modulus that was state dependent on contact pressure. Several models were developed using different methods. Hakiel [1] and Willett and Poesch [2] developed winding models based on theory of elasticity approaches. Pfeiffer [3] developed a winding model based on an energy method. Mollamahmutoglu and Good [4] based their formulation on the finite element method.

Pfeiffer [5] had noted a logarithmic behavior between pressure (P) and radial strain $\left(\varepsilon_{\mathrm{r}}\right)$ in a stack of web material in compression:

$$
P=K_{1}\left(e^{K_{2} \varepsilon_{r}}\right)
$$

He also noted the radial modulus $\left(E_{r}\right)$ was linearly dependent on pressure or radial stress:

$$
E_{r}=\frac{d P}{d \varepsilon_{r}}=K_{2}\left(P+K_{1}\right)=K_{2}\left(K_{1}-\sigma_{r}\right)
$$

In practice pressure and strain data are collected for a stack of the web material subject to normal compression. The factors $\mathrm{K}_{1}$ and $\mathrm{K}_{2}$ are varied until Equation $\{1\}$ agrees best with the collected data. The logarithmic variation noted by Pfeiffer is the result of multiple physical behaviors. One such behavior is the surface contact of webs in compression. Webs typically have various surface asperities that may either be inherent in the material or the result of the machinery on which the web was made. As the web is compressed the highest surface asperities first come into contact with asperities on the 
opposing web surface. With increased compression, the highest web asperities are further compressed and shorter asperities may initiate contact resulting in the logarithmic behavior. This is a source of geometric nonlinearity. Furthermore the asperities may suffer inelastic deformation due to large strains which results in material nonlinearity. Webs are often inhomogeneous and the geometric and material nonlinearity may also be occurring within the web due to internal contacts and void closure. All of the geometric and material nonlinearity become vested as a material nonlinearity that is characterized by the $K_{1}$ and $K_{2}$ parameters. The models of Hakiel [1], Willett et al [2] and Pfeiffer [3] determine the change in winding residual stresses due to the addition of a most recent layer. These changes in stresses are used to update the total stress in each layer. During solution the radial modulus is constant but unique to each layer. After the total pressure is known, the radial modulus (2) is updated prior to the addition and solution for the next layer. As noted earlier, winding tension is important in the development of residual stresses. A portion of this tension can be lost within the outer layer as it wound onto the roll resulting from radial deformation of the layers beneath, demonstrated by Good et. al. [6]. The winding model of Mollamahmutoglu and Good [4] accommodated this lost tension phenomena using a pre-stress boundary condition for the outer layer and a finite element formulation. They also pointed out that the previous methods of attack [1-3] were not rigorous nonlinear solutions. Their work accounted for the effects of large deformations and strains and utilized Newton Raphson iteration as each layer was added to the roll. This work demonstrated that very few iterations were needed to achieve convergence. The earlier models [1-3] were validated with laboratory winding tests where pressures within wound rolls were measured and compared with results from models. For the materials wound the agreement was good. This was evidence that the treatment of nonlinearity was adequate for those materials. Mollamahmutoglu and Good demonstrated good agreement between model and test results over a broad range of radial modulus associated with paper, tissue and non-woven webs. The $1 \mathrm{D}$ winding code developed herein will follow the finite element derivation of Mollamahmutoglu and Good [4]. That derivation will be expanded to encompass multilayer laminated materials.

Winding models have progressed to two dimensional (2D) axisymmetric developments that allow study of web thickness and length non-uniformity. Models progressed from pseudo 2D models composed of 1D sectors [7, 8] to 2D axisymmetric formulations [9,10]. Fully 2D axisymmetric formulations allow web length and thickness non-uniformities to affect the radius of the outer layer of the winding roll in the CD and interact with the allocation of web tension in the CD [11-13]. The development disclosed within this paper will be a plain strain 1D axisymmetric model but the findings will be applicable to 2D axisymmetric models.

\section{DEVELOPMENT}

\section{A 1D Plane Strain Orthotropic Finite Element Winding Model}

This development is a fundamental building block in the development of a laminate winding mode

l. The development begins with the selection of 1D shape functions in the natural coordinate $\xi$ shown in Figure 1:

$$
N_{i}=\frac{1-\xi}{2} \quad N_{j}=\frac{1+\xi}{2}
$$




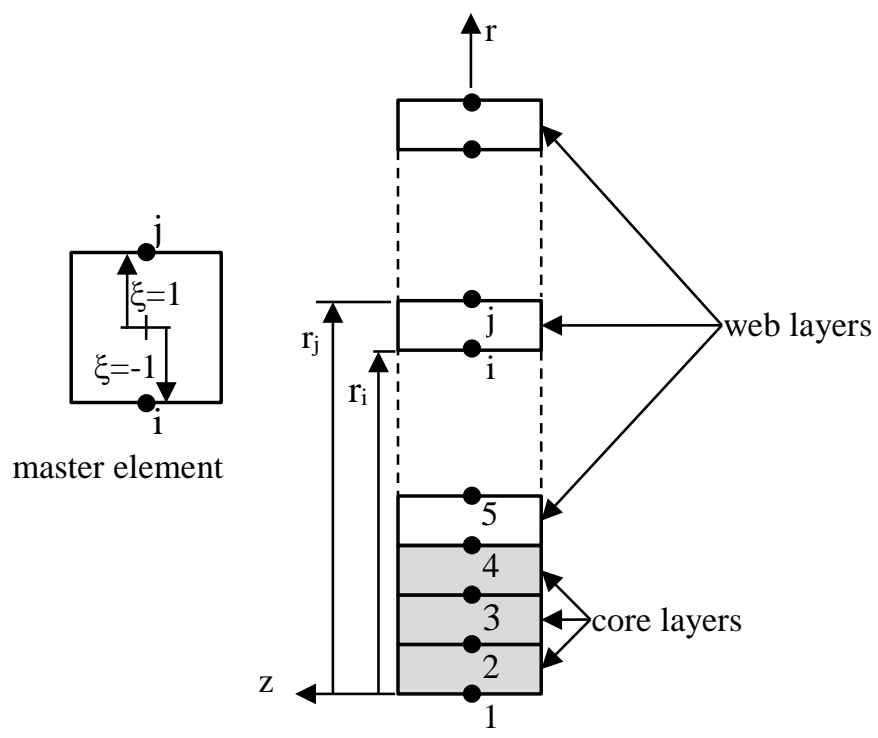

Figure 1 - 1D Axisymmetric Finite Element Model of Wound Roll

These shape functions will be used in an isoparametric formulation to interpolate the radial location (r) and the radial deformation (u) within a $1 \mathrm{D}$ axisymmetric finite element:

$$
\begin{aligned}
& r=\left[\begin{array}{ll}
N_{i} & N_{j}
\end{array}\right]\left\{\begin{array}{l}
r_{i} \\
r_{j}
\end{array}\right\} \\
& u=\left[\begin{array}{ll}
N_{i} & N_{j}
\end{array}\right]\left\{\begin{array}{l}
u_{i} \\
u_{j}
\end{array}\right\}
\end{aligned}
$$

Equation $\{4\}$ can be rearranged to produce a coordinate map equation relating the $\xi$ and $\mathrm{r}$ coordinates. Note that $r_{j}-r_{i}$ is the undeformed web thickness $h$ :

$$
\xi=\frac{2 r-\left(r_{i}+r_{j}\right)}{r_{j}-r_{i}}=\frac{2 r-\left(r_{i}+r_{j}\right)}{h}
$$

With the deformation (5) known the strains can be determined. In a 1D axisymmetric plane strain formulation the strains are:

$$
\varepsilon_{r}=\frac{d u}{d r}=\frac{d u}{d \xi} \frac{d \xi}{d r}=\left[\begin{array}{ll}
-\frac{1}{h} & \frac{1}{h}
\end{array}\right]\left\{\begin{array}{l}
u_{i} \\
u_{j}
\end{array}\right\}
$$

For purposes of stiffness development the tangential strain will be determined at the centroid of the finite element where $\bar{r}=\frac{r_{i}+r_{j}}{2}$ :

$$
\begin{gathered}
\varepsilon_{\theta}=\frac{u}{r}=\left[\begin{array}{ll}
\frac{N_{i}}{r} & \frac{N_{j}}{r}
\end{array}\right]\left\{\begin{array}{l}
u_{i} \\
u_{j}
\end{array}\right\}=\left[\begin{array}{ll}
\frac{1}{2 \bar{r}} & \frac{1}{2 \bar{r}}
\end{array}\right]\left\{\begin{array}{l}
u_{i} \\
u_{j}
\end{array}\right\} \\
\varepsilon_{z}=\gamma_{r z}=0
\end{gathered}
$$


The non-zero strains are thus:

$$
\left\{\begin{array}{l}
\varepsilon_{r} \\
\varepsilon_{\theta}
\end{array}\right\}=\left[\begin{array}{cc}
-\frac{1}{h} & \frac{1}{h} \\
\frac{1}{2 \bar{r}} & \frac{1}{2 \bar{r}}
\end{array}\right]\left\{\begin{array}{l}
u_{i} \\
u_{j}
\end{array}\right\}=[\bar{B}]\{u\}
$$

In cylindrical coordinates the elongating strains $(\varepsilon)$ and stresses $(\sigma)$ are related by the following constitutive relations. Note that Maxwell's relations requiring symmetry in the constitutive equations have been enforced:

$$
\left\{\begin{array}{l}
\varepsilon_{r} \\
\varepsilon_{\theta} \\
\varepsilon_{z}
\end{array}\right\}=\left[\begin{array}{ccc}
\frac{1}{E_{r}} & -\frac{v_{\theta r}}{E_{\theta}} & -\frac{v_{z r}}{E_{z}} \\
-\frac{v_{\theta r}}{E_{\theta}} & \frac{1}{E_{\theta}} & -\frac{v_{z \theta}}{E_{z}} \\
-\frac{v_{z r}}{E_{z}} & -\frac{v_{z \theta}}{E_{z}} & \frac{1}{E_{z}}
\end{array}\right]\left\{\begin{array}{l}
\sigma_{r} \\
\sigma_{\theta} \\
\sigma_{z}
\end{array}\right\}
$$

In plane strain $\varepsilon_{z}=0$ which allows the relation of the stresses from (11):

$$
\varepsilon_{z}=0=-\frac{v_{z r}}{E_{z}} \sigma_{r}-\frac{v_{z \theta}}{E_{z}} \sigma_{\theta}+\frac{\sigma_{z}}{E_{z}} \text { or } \sigma_{z}=v_{z r} \sigma_{r}+v_{z \theta} \sigma_{\theta}
$$

For the 1D axisymmetric plane strain model the non-zero strains become:

$$
\{\varepsilon\}=\left\{\begin{array}{l}
\varepsilon_{r} \\
\varepsilon_{\theta}
\end{array}\right\}=\left[\begin{array}{cc}
\frac{1}{E_{r}}-\frac{v_{z r}^{2}}{E_{z}} & -\frac{v_{\theta r}}{E_{\theta}}-\frac{v_{z r} v_{z \theta}}{E_{z}} \\
-\frac{v_{\theta r}}{E_{\theta}}-\frac{v_{z r} v_{z \theta}}{E_{z}} & \frac{1}{E_{\theta}}-\frac{v_{z \theta}^{2}}{E_{z}}
\end{array}\right]\left\{\begin{array}{l}
\sigma_{r} \\
\sigma_{\theta}
\end{array}\right\}=[D]^{-1}\{\sigma\}
$$

The constitutive relations [D] relate stress to strain and are needed in the development:

$$
\begin{gathered}
\{\sigma\}=[D]\{\varepsilon\}=[D][B]\{u\} \\
{[D]=\frac{E_{\theta}\left[\begin{array}{cc}
E_{r}\left(E_{\theta} v_{z \theta}^{2}-E_{z}\right) & -E_{r}\left(E_{\theta} v_{z r} v_{z \theta}+E_{z} v_{\theta r}\right) \\
-E_{r}\left(E_{\theta} v_{z r} v_{z \theta}+E_{z} v_{\theta r}\right) & E_{\theta}\left(E_{r} v_{z r}^{2}-E_{z}\right)
\end{array}\right]}{E_{z}\left(E_{r} v_{\theta r}^{2}-E_{\theta}\right)+E_{\theta}\left(E_{\theta} v_{z \theta}^{2}+E_{r} v_{z r}\left(v_{z r}+2 v_{z \theta} v_{\theta r}\right)\right)}=\left[\begin{array}{ll}
D_{11} & D_{12} \\
D_{21} & D_{22}
\end{array}\right]}
\end{gathered}
$$

The concept of pre-stress $\left(\sigma_{0}\right)$ and pre-strain $\left(\varepsilon_{0}\right)$ is often used in finite element derivations to accommodate thermal stress and strain. This concept will be employed here to introduce the MD stress in the web due to web tension in the winder tension zone. The strain energy in a finite element $(\mathrm{U})$ is:

$$
U_{e}=\frac{1}{2} \int_{0}^{2 \pi} \int_{A}\{\sigma\}^{T}\{\varepsilon\} r d A d \theta-\int_{0}^{2 \pi} \int_{A}\{\sigma\}^{T}\left\{\varepsilon_{o}\right\} r d A d \theta
$$

Substituting the developed representations for stress and stain yields:

$$
U_{e}=\frac{2 \pi}{2}\{q\}^{T} \int_{A}\{\bar{B}\}^{T}[D]\{\bar{B}\} \bar{r} d A\{q\}-2 \pi\{q\}^{T} \int_{A}\{\bar{B}\}^{T}[D]\left\{\varepsilon_{o}\right\} \bar{r} d A
$$

The element stiffness matrix $\left[\mathrm{K}_{\mathrm{e}}\right]$ is integral to the $1^{\text {st }}$ term in Equation $\{17\}$ :

$$
U_{e}=\frac{1}{2}\{q\}^{T}\left[K_{e}\right]\{q\}-\{q\}^{T}\left\{f_{e}\right\}
$$




$$
\left[K_{e}\right]=2 \pi \int_{A}\{\bar{B}\}^{T}[D]\{\bar{B}\} \bar{r} d A=2 \pi \bar{r} A_{e}\{\bar{B}\}^{T}[D]\{\bar{B}\}=2 \pi \bar{r} h W\{\bar{B}\}^{T}[D]\{\bar{B}\}
$$

where $W$ is the web width. Substituting Equations $\{10\}$ and $\{15\}$ into $\{19\}$ yields:

$$
\left[K_{e}\right]=\left[\begin{array}{cc}
\frac{\pi W}{2}\left(\frac{4 \bar{r}}{h} D_{11}+\frac{h}{\bar{r}} D_{22}-4 D_{12}\right) & r W\left(\frac{h}{2 \bar{r}} D_{22}-\frac{2 \bar{r}}{h} D_{11}\right) \\
\pi W\left(\frac{h}{2 \bar{r}} D_{22}-\frac{2 \bar{r}}{h} D_{11}\right) & \frac{\pi W}{2}\left(\frac{4 \bar{r}}{h} D_{11}+\frac{h}{\bar{r}} D_{22}-4 D_{12}\right)
\end{array}\right]=\left[\begin{array}{cc}
k_{11} & k_{12} \\
k_{21} & k_{22}
\end{array}\right]
$$

The force vector $\left\{\mathrm{f}_{\mathrm{e}}\right\}$ is integral to the $2^{\text {nd }}$ term in Equation $\{18\}$ :

$$
f_{e}=2 \pi \int_{A}\{\bar{B}\}^{T}[D]\left\{\varepsilon_{o}\right\} \bar{r} d A=2 \pi \bar{r} h W\{\bar{B}\}^{T}[D]\left\{\varepsilon_{o}\right\}=2 \pi \bar{r} h W\{\bar{B}\}^{T}\left\{\sigma_{o}\right\}
$$

Substituting Equations $\{10\}$ and $\{15\}$ into $\{21\}$ yields:

$$
\left\{f_{e}\right\}=2 \pi \bar{r} h W\left\{\begin{array}{l}
\frac{\sigma_{\theta}}{2 \bar{r}}-\frac{\sigma_{r}}{h} \\
\frac{\sigma_{\theta}}{2 \bar{r}}+\frac{\sigma_{r}}{h}
\end{array}\right\}_{0}
$$

The only pre-stress in the outer layer is the tangential stress $\left(\sigma_{\theta}\right)$ which is equivalent to the web stress due to web tension $\left(\mathrm{T}_{\mathrm{w}}\right)$ and there is no radial pre-stress component $\left(\sigma_{\mathrm{r}}\right)$. Also a tensile $\sigma_{\theta}$ stress in the outer lap would produce forces in a positive r direction at nodes $i$ and $j$ that would result in a negative contact pressure between the outer layer and the layer beneath. Thus a negative value of web stress is substituted into Equation $\{22\}$ and the force vector reduces to:

$$
\left\{f_{e}\right\}=-\pi h W T_{w}\left\{\begin{array}{l}
1 \\
1
\end{array}\right\}
$$

The winding tension $\left(\mathrm{T}_{\mathrm{w}}\right)$ in Equation $\{23\}$ can take any form as a function of wound roll radius chosen; herein a constant value of winding tension was selected. With a developed stiffness matrix and force vector the development of the finite element formulation is near complete. The stiffness matrix $\{20\}$ can be used recursively to develop element stiffness matrices for the core and for the layers of web material added to the core. An example is shown in Equation $\{24\}$ in which the changes in deformation $\left(\delta u_{i}\right)$ are being sought as a result of accreting the third web layer. Note the core is being crudely modeled here with 2 axisymmetric elements, in most cases 5 core layers has been found sufficient to model the core accurately. Each web layer is modeled with 1 axisymmetric element, which is sufficient.

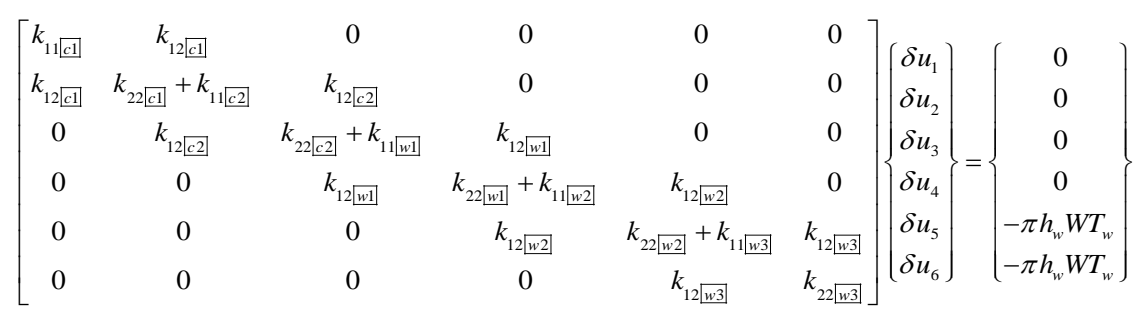

The assembly of the stiffness matrices begins with assembling all the core matrices. Several elements should be used to model the core which is usually considerably thicker 
than the web. Since the finite element will allow at best constant values of stress within the domain of the element, several elements are needed to properly characterize the mechanical behavior of the core. The accretive solution begins with one web layer being added sequentially. Note that one web and two web layer solutions had to precede that shown in Equation $\{24\}$ such that the $\mathrm{D}_{\mathrm{ij}}$ terms in $\{15\}$ were known for the web layers that depend on the state dependent radial modulus that varies with pressure. The changes in deformation that result from solving the independent set of equations such as those shown in Equation $\{24\}$ can then be used to determine the increments in stress within each element due to the addition of the most recent layer. Such a computation is shown here for element W2:

$$
\left\{\delta \sigma_{\underline{\underline{W 2}}}\right\}=\left\{\begin{array}{l}
\delta \sigma_{r} \\
\delta \sigma_{\theta}
\end{array}\right\}=[D]_{[\underline{W 2}}[\bar{B}]_{\underline{\underline{W 2}}}\left\{\begin{array}{l}
\delta u_{4} \\
\delta u_{5}
\end{array}\right\}
$$

Equation $\{12\}$ can then be used to determine the change in axial stress $\left(\delta \sigma_{z}\right)$ in element W2:

$$
\delta \sigma_{z}=v_{z r} \delta \sigma_{r}+v_{z \theta} \delta \sigma_{\theta}
$$

Changes in stresses would be calculated for each element of the core and for all layers in the wound roll. The total stresses in a particular layer are determined by summing all the changes in stress in that layer from the point when that layer was added until the most recent layer $n$ was accreted on the wound roll. For layer W2:

$$
\{\sigma\}_{\underline{W 2}}=\left\{\begin{array}{c}
\sum_{i=2}^{n} \delta \sigma_{r i[\underline{W 2}} \\
\sum_{i=2}^{n} \delta \sigma_{\theta i[\underline{W 2}}+T_{w} \\
\sum_{i=2}^{n} \delta \sigma_{z i[W 2}
\end{array}\right\}=\left\{\begin{array}{c}
\sigma_{r} \\
\sigma_{\theta} \\
\sigma_{z}
\end{array}\right\}^{W 2}
$$

The total pressure in layer $\mathrm{W} 2$ is now known $\left(\mathrm{P}=-\sigma_{\mathrm{r}}\right)$ and can be used to update the radial modulus $\left(E_{r}\right)$ using Equation $\{2\}$ for this element. The stiffness matrix for element W2 can then be updated using Equations $\{15\}$ and $\{20\}$. These calculations in Equation $\{27\}$ are repeated for all $n$ layers in the wound roll. Then a new set of equations similar to those shown in $\{25\}$ is formed to solve for the differential displacements throughout the wound roll due to the addition of the $n+1$ layer. The differential displacements for each node can be summed to determine the total deformation of each node due to all the layers added outside of a given node. Equations similar to \{24\} through \{27\} are assembled repeatedly and solved until a defined number of layers are wound onto the core or a defined outer roll radius is achieved.

Validation: 1D winding models have been verified at various levels [1-4]. The interlayer pressure can be measured using steel shim on narrow rolls quite accurately. The steel shim is often enveloped in brass shim and wound into rolls. The pull force required to induce slip between the steel and brass shim is related to the pressure between layers in the wound roll. The relationship is best obtained by inserting these pressure transducers into a stack of the web material to be wound. A material testing system is 
used to subject the stack to various pressures and the force required to induce slip is measured at each stack test pressure.

The model developed herein will be verified for a newsprint web with the properties shown in Table 1. Note that orthotropic property input is possible for both the web and the core.

\begin{tabular}{|c|c|c|c|}
\hline Web Thickness (mm) & 0.071 & Core Inner Radius (cm) & 3.81 \\
\hline Finish Radius (cm) & 13.35 & Core Outer Radius (cm) & 4.45 \\
\hline \multicolumn{2}{|c|}{ Web Width $(\mathrm{cm})$} & \multicolumn{2}{|l|}{15.26} \\
\hline \multicolumn{2}{|c|}{ Winding Stress (MPa) } & \multicolumn{2}{|l|}{5.17} \\
\hline \multicolumn{2}{|c|}{ Web Properties } & \multicolumn{2}{|c|}{ Core Properties } \\
\hline $\mathrm{E}_{\theta}=\mathrm{E}_{\mathrm{z}}(\mathrm{MPa})$ & 3370 & $\mathrm{E}_{\mathrm{r}}=\mathrm{E}_{\theta}=\mathrm{E}_{\mathrm{z}}(\mathrm{GPa})$ & 200 \\
\hline $\mathrm{K}_{1}(\mathrm{KPa})$ & 1.175 & $v_{\theta r}=v_{z r}=v_{z \theta}$ & 0.3 \\
\hline $\mathrm{K}_{2}$ & 45.14 & & \\
\hline$v_{\theta r}=v_{z r}=v_{z \theta}$ & 0.3 & & \\
\hline
\end{tabular}

Table 1 - Winding, Web and Core Properties for Winding Newsprint

The results of the verification tests are shown in Figure 2. The test data points are the average pressure measurements from 3 winding tests where pull tab pressure transducers were wound into the rolls consistently at the wound roll radial positions shown in the charts. The error bars show the standard deviation of the data at each radius. Model results are shown for both plane stress and plane strain material behaviors. The plane strain model developed herein can produce plane stress behavior if $v_{\mathrm{zr}}$ and $v_{\mathrm{z} \theta}$ are set to zero. In general the comparison of model results with tests is very good with the test results comparing somewhat better with the plane stress model behavior. The web width may have been inadequate to achieve the plane strain behavior. Note the tangential stresses $\left(\sigma_{\theta}\right)$ resulting from the two material behaviors are essentially equal. Substantial negative axial stresses can be developed when plane strain behaviors are achieved. In plane strain conditions the axial stresses tend to vanish at the outer lap and in this case near the core which was axially much stiffer than the web in this example. 

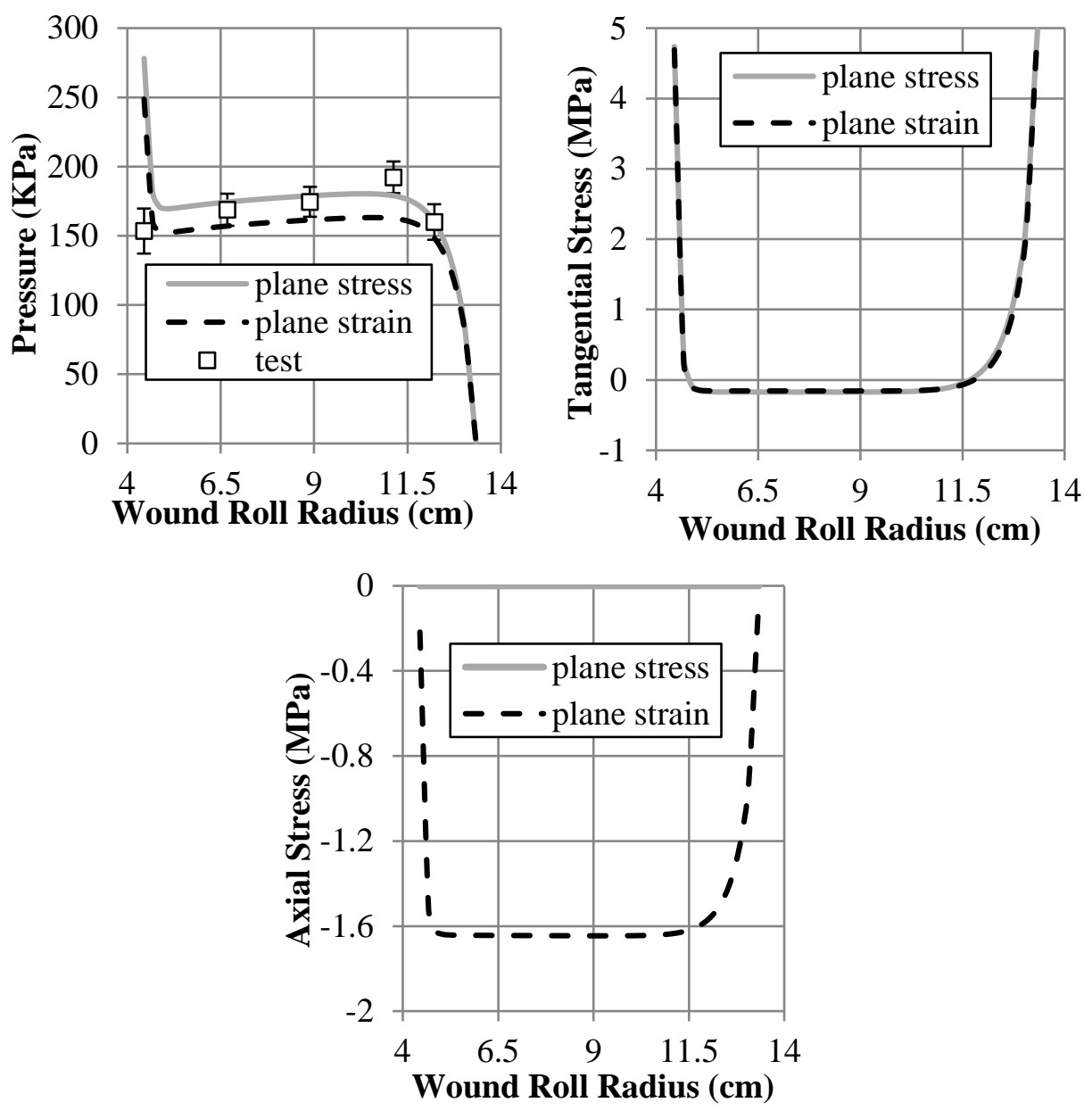

Figure 2 - Verification of Orthotropic Winding Model on Newsprint

\section{A 1D Plane Strain Orthotropic Laminate Finite Element Winding Model}

A stiffness matrix and force vector for a plane strain homogenous web was developed in Equations $\{20\}$ and $\{23\}$, respectively. Those developments will be extended to a two layer laminate web. It will be assumed that the agent used to bond the layers together in the laminator does not contribute to the stiffness of the laminate. It will also be assumed that the behavior of a stack of laminates in compression will be characterized in a compression test similar to that described in the Background. 


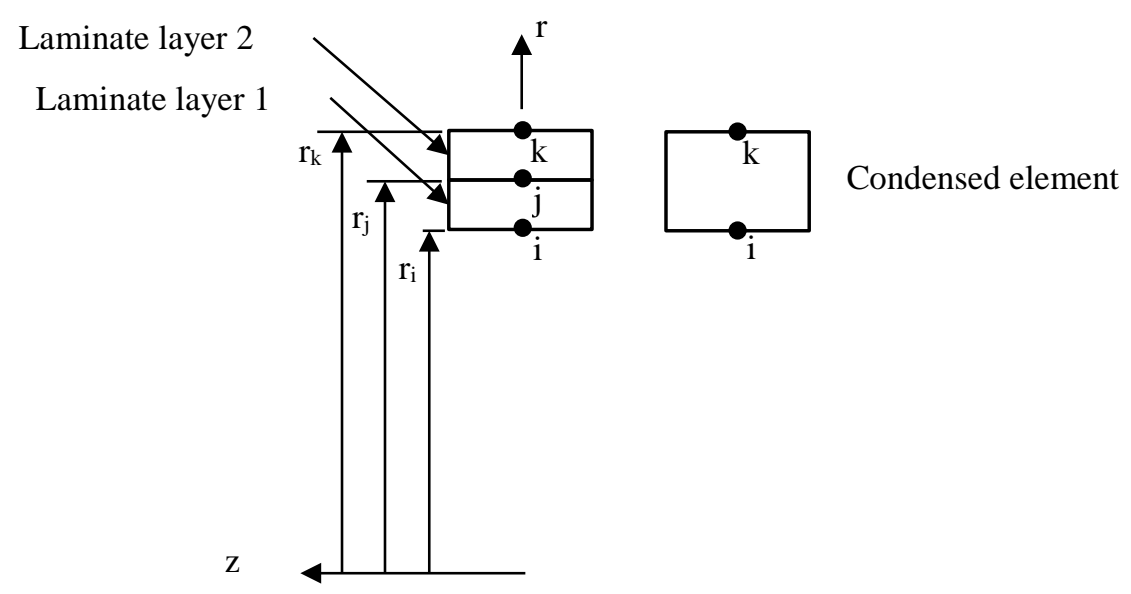

Figure 3 - A 1D Axisymmetric Laminate Finite Element

A laminate is now accreted to the winding roll and a stiffness matrix and a force vector for the laminate is needed. Equations $\{20\}$ and $\{23\}$ can be used to determine the stiffness and forces the two layers (1 and 2$)$ in the laminate:

$$
K_{(1)}=\left[\begin{array}{ll}
K_{1 i i} & K_{1 i j} \\
K_{1 i j} & K_{1 j j}
\end{array}\right], f_{(1)}=\left\{\begin{array}{l}
f_{1 i} \\
f_{1 j}
\end{array}\right\} \text { and } K_{(2)}=\left[\begin{array}{ll}
K_{2 j j} & K_{2 j k} \\
K_{2 j k} & K_{2 k k}
\end{array}\right], f_{(2)}=\left\{\begin{array}{c}
f_{2 j} \\
f_{2 k}
\end{array}\right\}
$$

where $i, j$ and $k$ refer to the nodes in Figure 3. Since the two layers have node $j$ in common the direct stiffness assembly method can be used to combine the stiffness matrices and force vectors:

$$
K_{\text {Laminate }}=\left[\begin{array}{ccc}
K_{1 i i} & K_{1 i j} & 0 \\
K_{1 i j} & K_{1 j j}+K_{2 j j} & K_{2 j k} \\
0 & K_{2 j k} & K_{2 k k}
\end{array}\right] \text { and } f_{\text {Laminate }}=\left\{\begin{array}{c}
f_{1 i} \\
f_{1 j}+f_{2 j} \\
f_{2 k}
\end{array}\right\}
$$

where:

$$
\begin{array}{cc}
K_{1 i i}=\left.\frac{\pi}{2} w\left(\frac{4 \bar{r}}{h} D_{11}+\frac{h}{\bar{r}} D_{22}-4 D_{12}\right)\right|_{(1)} & K_{2 j j}=\left.\frac{\pi}{2} w\left(\frac{4 \bar{r}}{h} D_{11}+\frac{h}{\bar{r}} D_{22}-4 D_{12}\right)\right|_{(2)} \\
K_{1 i j}=\left.\pi w\left(\frac{h}{2 \bar{r}} D_{22}-\frac{2 \bar{r}}{h} D_{11}\right)\right|_{(1)} & K_{2 j k}=\left.\pi w\left(\frac{h}{2 \bar{r}} D_{22}-\frac{2 \bar{r}}{h} D_{11}\right)\right|_{(2)} \\
K_{1 j j}=\left.\frac{\pi}{2} w\left(4 D_{12}+\frac{h}{\bar{r}} D_{22}+\frac{4 \bar{r}}{h} D_{12}\right)\right|_{(1)} & K_{2 k k}=\left.\frac{\pi}{2} w\left(4 D_{12}+\frac{h}{\bar{r}} D_{22}+\frac{4 \bar{r}}{h} D_{12}\right)\right|_{(2)}
\end{array}
$$

and:

$$
f_{(1)}=\left\{\begin{array}{l}
f_{1 i} \\
f_{1 j}
\end{array}\right\}=-\left.\pi h W T_{w}\right|_{(1)}\left\{\begin{array}{l}
1 \\
1
\end{array}\right\} \text { and } f_{(2)}=\left\{\begin{array}{c}
f_{2 j} \\
f_{2 k}
\end{array}\right\}=-\left.\pi h W T_{w}\right|_{(2)}\left\{\begin{array}{l}
1 \\
1
\end{array}\right\}
$$

The subscripts (1) and (2) in Equations \{30\} and \{31\} denote stiffness and force terms associated with layers 1 and 2 in the laminate. The stresses in layers 1 and 2 will be 
unique and will depend on conditions at the laminator which will be discussed later. An accretive solution similar to that shown for the single orthotropic layer in Equation \{24\} can now be developed and solved for the changes in radial deformation of the nodes $\left(\delta \mathrm{u}_{\mathrm{i}}\right)$. Those nodal changes in deformation can then be used to solve for changes in stress in all layers using Equation $\{25\}$.

Numerical Oscillation: When solutions were attempted of the laminate winding model described numerical oscillations were witnessed in some cases in the stresses output. In other cases the solution of the set of equations was not possible. If identical material properties were input for the two layers of the laminate, the oscillations vanished and solution of the sets of equations was always possible. The problem stemmed from the assumption that both layers of the laminate shared an identical Equation $\{2\}$ for the radial modulus $\left(\mathrm{E}_{\mathrm{r}}\right)$. The problem was solved using a condensation method.

Condensation: The equilibrium of the two-layer laminate can be stated as:

$$
\left[\begin{array}{ccc}
K_{1 i i} & K_{1 i j} & 0 \\
K_{1 j j} & K_{1 j j}+K_{2 j j} & K_{2 j k} \\
0 & K_{2 j k} & K_{2 k k}
\end{array}\right]\left\{\begin{array}{l}
\delta u_{i} \\
\delta u_{j} \\
\delta u_{k}
\end{array}\right\}=\left\{\begin{array}{c}
f_{1 i} \\
f_{1 j}+f_{2 j} \\
f_{2 k}
\end{array}\right\}
$$

The condensation method will be used to remove the internal degree of freedom at node $j$. These equations from Equation $\{32\}$ can be re-ordered as follows:

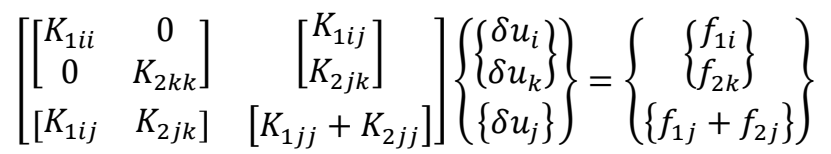

This can be rewritten symbolically as:

$$
\left[\begin{array}{ll}
{\left[K_{r r}\right]} & {\left[K_{r c}\right]} \\
{\left[K_{c r}\right]} & {\left[K_{c c}\right]}
\end{array}\right]\left\{\begin{array}{l}
\left\{\delta u_{r}\right\} \\
\left\{\delta u_{c}\right\}
\end{array}\right\}=\left\{\begin{array}{c}
\left\{r_{r}\right\} \\
\left\{r_{c}\right\}
\end{array}\right\}
$$

The condensed stiffness matrix is:

$$
K_{\text {condensed }}=\left[K_{r r}\right]-\left[K_{r c}\right]\left[K_{c c}\right]^{-1}\left[K_{c r}\right]=\left[\begin{array}{cc}
K_{1 i i}-\frac{K_{1 i j}^{2}}{K_{1 j j}+K_{2 j j}} & \frac{-K_{1 i j} K_{2 j k}}{K_{1 j j}+K_{2 j j}} \\
\frac{-K_{1 i j} K_{2 j k}}{K_{1 j j}+K_{2 j j}} & K_{2 k k}-\frac{K_{2 j k}^{2}}{K_{1 j j}+K_{2 j j}}
\end{array}\right]
$$

and the condensed force vector is:

$$
f_{\text {condensed }}=\left\{r_{r}\right\}-\left[K_{r c}\right]\left[K_{c c}\right]^{-1}\left\{r_{c}\right\}=\left\{\begin{array}{l}
f_{1 i}-\frac{K_{1 i j}}{K_{1 j j}+K_{2 j j}}\left(f_{1 j}+f_{2 j}\right) \\
f_{2 k}-\frac{K_{2 j k}}{K_{1 j j}+K_{2 j j}}\left(f_{1 j}+f_{2 j}\right)
\end{array}\right\}
$$

The condensed stiffness matrix and force vector can now be used in an accretive solution identical to that posed earlier for accreting single layers of web as given in Equation \{24\}. After solving for the changes in deformation due to a new outer laminate the changes in stress, the total stresses and the radial modulus in each layer must be updated. To 
compute the changes in stress requires the recovery of the deformation associated with the internal node $j$ in each condensed laminate element. That deformation can be recovered using:

$$
\begin{aligned}
& \left\{\delta u_{c}\right\}=\left[K_{c c}\right]^{-1}\left(\left[K_{c r}\right]\left\{\delta u_{r}\right\}-\left\{r_{c}\right\}\right) \\
& \left\{\delta u_{j}\right\}=\left[K_{1 j j}+K_{2 j j}\right]^{-1}\left(\left[\begin{array}{ll}
K_{1 i j} & K_{2 j k}
\end{array}\right]\left\{\begin{array}{l}
\delta u_{i} \\
\delta u_{k}
\end{array}\right\}-\left\{f_{1 j}+f_{2 j}\right\}\right)
\end{aligned}
$$

Now Equation $\{25\}$ can be used to determine the changes in stress in each layer of all the laminate that has been wound onto the roll. The total stresses are obtained using Equation $\{27\}$ but the winding stress in each layer of the laminate will be unique $\left(\mathrm{T}_{\mathrm{w} 1}\right.$ or $\left.\mathrm{T}_{\mathrm{w} 2}\right)$.

\section{Strain Matched versus Non Strain Matched Laminating Conditions}

Laminates are often strain matched at the site of lamination. Laminated webs that are not strain matched will curl when cut into discrete products which is often undesirable. It is not always possible to set the web tensions in the layers entering the laminator to achieve strain matching. Nonetheless the laminate must be wound and the winding tension in the laminate layers are important input with regard to the winding residual stresses. To achieve strain matching requires the web stress in each layer to be controlled prior to lamination according to the MD modulus of that layer:

$$
\varepsilon_{M D}=\frac{T_{w 1}}{E_{M D 1}}=\frac{T_{w 2}}{E_{M D 2}}
$$

The web layer tensions prior to lamination should be in equilibrium with the tension $T$ in the laminated web where $A_{1}$ and $A_{2}$ are the cross sectional areas of layers 1 and 2, respectively:

$$
T=T_{w 1} A_{1}+T_{w 2} A_{2}
$$

The total tension $T$ in the laminate can vary depending on the tension zone in the web line. In the winder tension zone if the total tension is T, Equations $\{35\}$ and $\{36\}$ can be used to determine the winding stress in each layer of the laminate:

$$
T_{w 1}=\frac{T E_{M D 1}}{E_{M D 1} A_{1}+E_{M D 2} A_{2}} \text { and } T_{w 2}=\frac{T E_{M D 2}}{E_{M D 1} A_{1}+E_{M D 2} A_{2}}
$$

In non-strain matched conditions the web layer tensions are set independently ( $\mathrm{T}_{\text {layer1 }}$ and $\mathrm{T}_{\text {layer2}}$ ) upstream of the laminator. Although strain matching the layers is desirable when considering curl defects it is not always possible to transport webs upstream of the laminator at tensions that would be required to strain match the two webs. It is assumed the total web tension may differ from the exit of the laminator to the entry of the winder. If the laminate web tension at the entry to the winder is $\mathrm{T}$, the winding tension for layers 1 and 2 will be:

$$
T_{1}=\frac{T_{\text {layer } 1}}{T_{\text {layer } 1}+T_{\text {layer } 2}} * T \text { and } T_{2}=\frac{T_{\text {layer } 2}}{T_{\text {layer } 1}+T_{\text {layer } 2}} * T
$$

\section{$\underline{\text { Lab Test Verification }}$}

The Strain Matched Condition at Lamination: A strain matched 2-layer laminate was used in winding tests to verify the model. The laminate is composed of a paper layer 
and an oriented polypropylene polymer layer. The inputs provided to the laminate winding code are shown in Table 2, the polypropylene is layer $i$ and paper is layer $j$. All input was measured except for the Poisson ratio terms which were assumed.

\begin{tabular}{|c|c|}
\hline Core inner radius & $0.0381 \mathrm{~m}(1.5 \mathrm{in})$ \\
\hline Core outer radius & $0.0445 \mathrm{~m}(1.75 \mathrm{in})$ \\
\hline Roll final radius & $0.1334 \mathrm{~m}(5.25 \mathrm{in})$ \\
\hline$E_{w \theta i}, E_{w z i}$ & $1.96 \mathrm{GPa}(285,188 \mathrm{psi})$ \\
\hline$E_{w \theta j}, E_{w z j}$ & $14.41 \mathrm{GPa}(2,091,000 \mathrm{psi})$ \\
\hline Web: $v_{\theta \mathrm{ri}}, v_{\mathrm{zri}}, v_{\mathrm{z} \theta \mathrm{i}}, v_{\theta \mathrm{r}}, v_{\mathrm{zr}}, v_{\mathrm{z} \theta \mathrm{j}}$ & 0.3 \\
\hline $\mathrm{E}_{\mathrm{cr}}, \mathrm{E}_{c q}, \mathrm{E}_{c z}$ & $206.7 \mathrm{GPa}(30 \mathrm{Mpsi})$ \\
\hline$v_{\theta \mathrm{rc}}, v_{\mathrm{zrc}}, v_{\mathrm{z} \theta \mathrm{c}}$ & 0.3 \\
\hline Web width w & $0.1524 \mathrm{~m}(6 \mathrm{in})$ \\
\hline Thickness $h_{i}$ and $h_{j}$ & $66.04 \mu \mathrm{m}(0.0026 \mathrm{in}), 53.34 \mu \mathrm{m}(0.0021$ \\
\hline$E_{r}\left(K_{1}, K_{2}\right)$ & in) \\
\hline$T$, winding tension & $9.03 \mathrm{KPa}(1.31 \mathrm{psi}), 71.1$ \\
\hline
\end{tabular}

Table 2 - Input for Laminate Winding Model - Strain Matched Case

Results for the strain matched case are shown in Figure 4. This is a narrow web which has not achieved plane strain conditions. The model result shown in Figure 4 is for the plane stress case which was achieved by input of zero for the Poisson ratios $v_{\mathrm{zri}}, v_{\mathrm{z} \theta \mathrm{i}}, v_{\mathrm{zr}}$, and $v_{\mathrm{z} \theta \mathrm{j}}$. When winding laminates there is a choice of which layer faces the outside of the roll. The model shows no effect whether the paper or the polypropylene is chosen for layers $i$ or $j$. The winding tests were conducted with the paper facing outwards three times and then with the polypropylene facing outward three times. The test data points in Figure 4 are the average of three pressure measurements taken with pull tabs and the error bars indicate the standard deviation of the data. Use of the statistical t-test indicated that the data taken with the paper facing outward could not be claimed different than the data collected when the polypropylene faced outward. The agreement between the model for plane stress conditions and the test data is good. The tangential stress results show that the paper, whose in-plane modulus was roughly 7 times larger than that of the polypropylene, bore significantly larger stress at the outside of the roll. This was expected as a result of the strain matched condition. The tangential stresses in the outer layer produce a total laminate tension slightly less than the winding tension:

$$
\begin{aligned}
\left(\sigma_{\theta i} h_{i}+\sigma_{\theta j} h_{j}\right) W & =(0.43 M P a * 66.04 \mu m+3.13 M P a * 53.34 \mu m) 0.1524 m \\
& =29.8 N \approx T
\end{aligned}
$$

This is expected as a result of the tension loss phenomena where the laminate layers beneath the outer layer have deformed inward due to the pressure induced by the outer layer. 

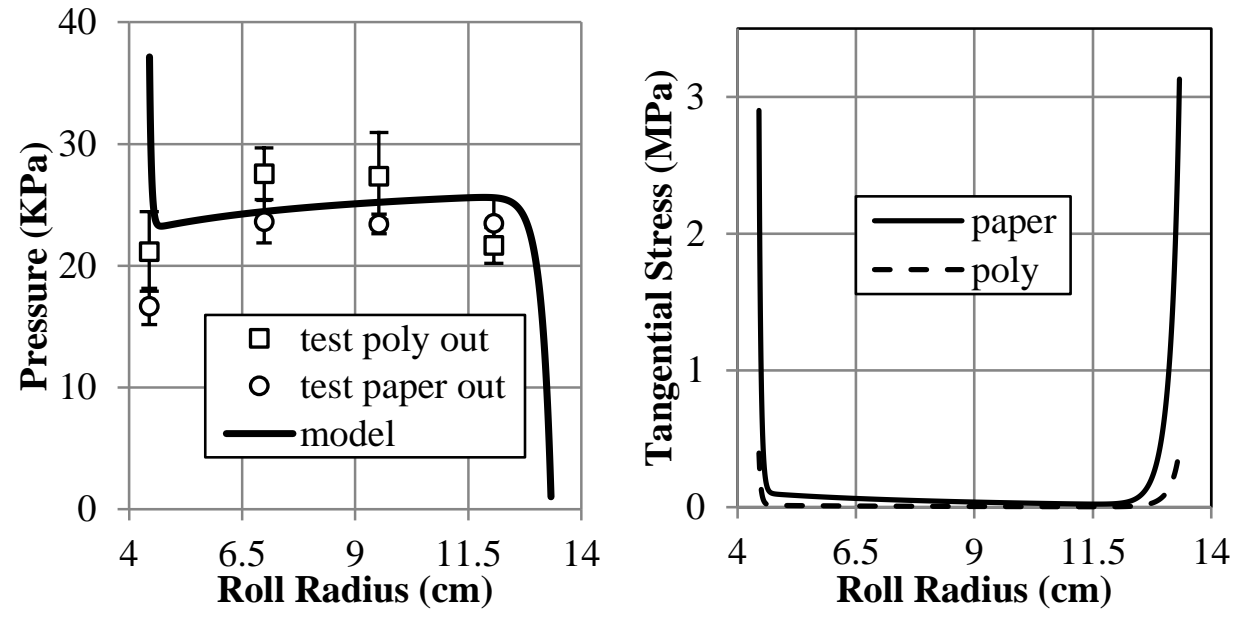

Figure 4 - Strain Matched Case T=2.1 N/cm

\section{The Non Strain Matched Condition at Lamination}

These tests were conducted on a laminating process web line. Layers $i$ and $j$ are a paper and a polypropylene web in these tests, respectively. The winding and material parameters are provided in Table 3.

\begin{tabular}{|c|c|c|}
\hline Core inner radius & \multicolumn{2}{|c|}{$0.089 \mathrm{~m}$ (3.5 in) } \\
\hline Core outer radius & \multicolumn{2}{|c|}{$0.105 \mathrm{~m}$ (4.15 in) } \\
\hline Roll final radius & \multicolumn{2}{|c|}{$0.2517 \mathrm{~m}(9.91 \mathrm{in})$} \\
\hline$E_{w \theta i}, E_{w z i}$ & \multicolumn{2}{|c|}{$6.12 \mathrm{GPa}(887,600 \mathrm{psi})$} \\
\hline$E_{w \theta j}, E_{w z j}$ & \multicolumn{2}{|c|}{$2.07 \mathrm{GPa}(300,000 \mathrm{psi})$} \\
\hline Web: $v_{\theta \mathrm{ri}}, v_{\mathrm{zri}}, v_{\mathrm{z} \theta \mathrm{i}}, v_{\theta \mathrm{rj}}, v_{\mathrm{zrj}}, v_{\mathrm{z} \theta \mathrm{j}}$ & \multicolumn{2}{|c|}{0.3} \\
\hline $\mathrm{E}_{c r}, \mathrm{E}_{c q}, \mathrm{E}_{c z}$ & \multicolumn{2}{|c|}{ 68.9 GPa (10 Mpsi) } \\
\hline$v_{\theta \mathrm{rc}}, v_{\mathrm{zrc}}, v_{\mathrm{z} \theta \mathrm{c}}$ & \multicolumn{2}{|c|}{0.3} \\
\hline Web width w & \multicolumn{2}{|c|}{$0.6858 \mathrm{~m}$ (27 in) } \\
\hline Thickness $h_{i}$ and $h_{j}$ & \multicolumn{2}{|c|}{$\begin{array}{c}55.88 \mu \mathrm{m}(0.0022 \text { in }), 66.04 \mu \mathrm{m}(0.0026 \\
\text { in) }\end{array}$} \\
\hline \multirow[t]{2}{*}{$E_{r}\left(K_{1}, K_{2}\right)$} & \multicolumn{2}{|c|}{$1.01 \mathrm{KPa}(0.146 \mathrm{psi}), 117.1$} \\
\hline & Case A & Case B \\
\hline Laminating Tension i & $302.5 \mathrm{~N}(68 \mathrm{lb})$ & $355.9 \mathrm{~N}(80 \mathrm{lb})$ \\
\hline Laminating Tension $\mathrm{j}$ & $89.0 \mathrm{~N} \mathrm{(20} \mathrm{lb)}$ & $44.5 \mathrm{~N}(10 \mathrm{lb})$ \\
\hline$T$, winding tension & \multicolumn{2}{|c|}{$95.6 \mathrm{~N}(21.5 \mathrm{lb})$} \\
\hline
\end{tabular}

Table 3 - Input for Laminate Winding Model: Non-Strain Matched Case

In this process machine the web tension downstream of the laminator differs from the laminate tension in the winder tension zone. Equation $\{38\}$ was employed to determine the tensions in the laminate layers in the winding model. Shim pressure transducers were wound into the edge of the winding roll as shown in Figure 5. 


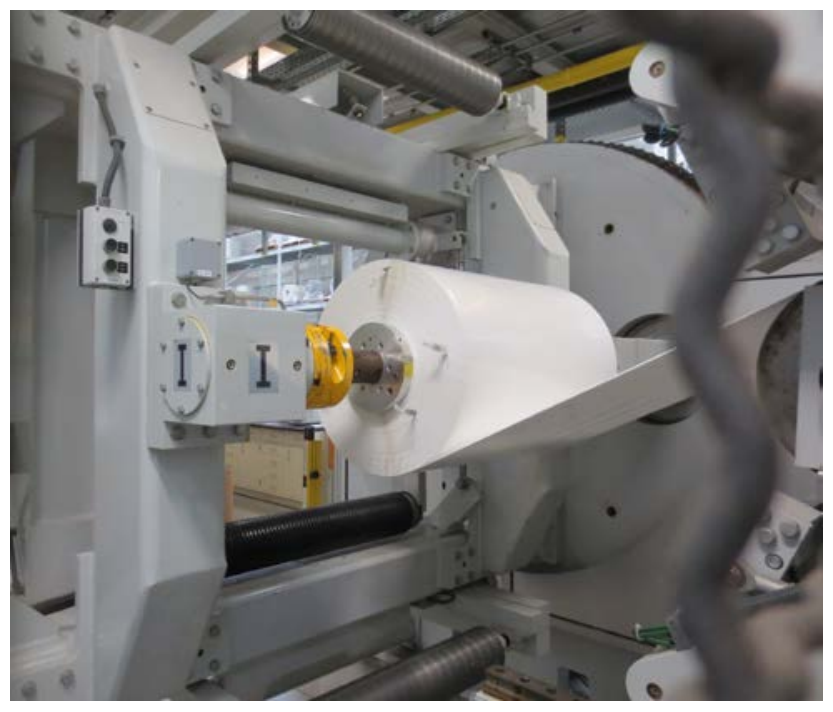

Figure 5 - Machine Set Up for Laminate Web Winding Tests

Results are shown in Figure 6 for both plane stress and plane strain material behaviors. These tests were conducted 3 times and the test data in Figure 6 represent the average of the pressure measurements at each radial location. The height of the error bars represents the standard deviation of the data. The test pressures agree best with the plane stress case but the plane strain results agree reasonably well too. Tangential and axial stresses throughout the roll are shown as well for both material behaviors.

Results are also shown in Figure 7 for a Case B where the tensions at the laminator were set markedly different from Case A but the tension in the laminated web at the winder was the same.

Note the model shows no difference in pressure for Cases A and B. This indicates pressure is being affected by total winding tension and not the laminating tensions. The tangential stresses are affected by the lamination tensions and although not shown here by winding tension too. 

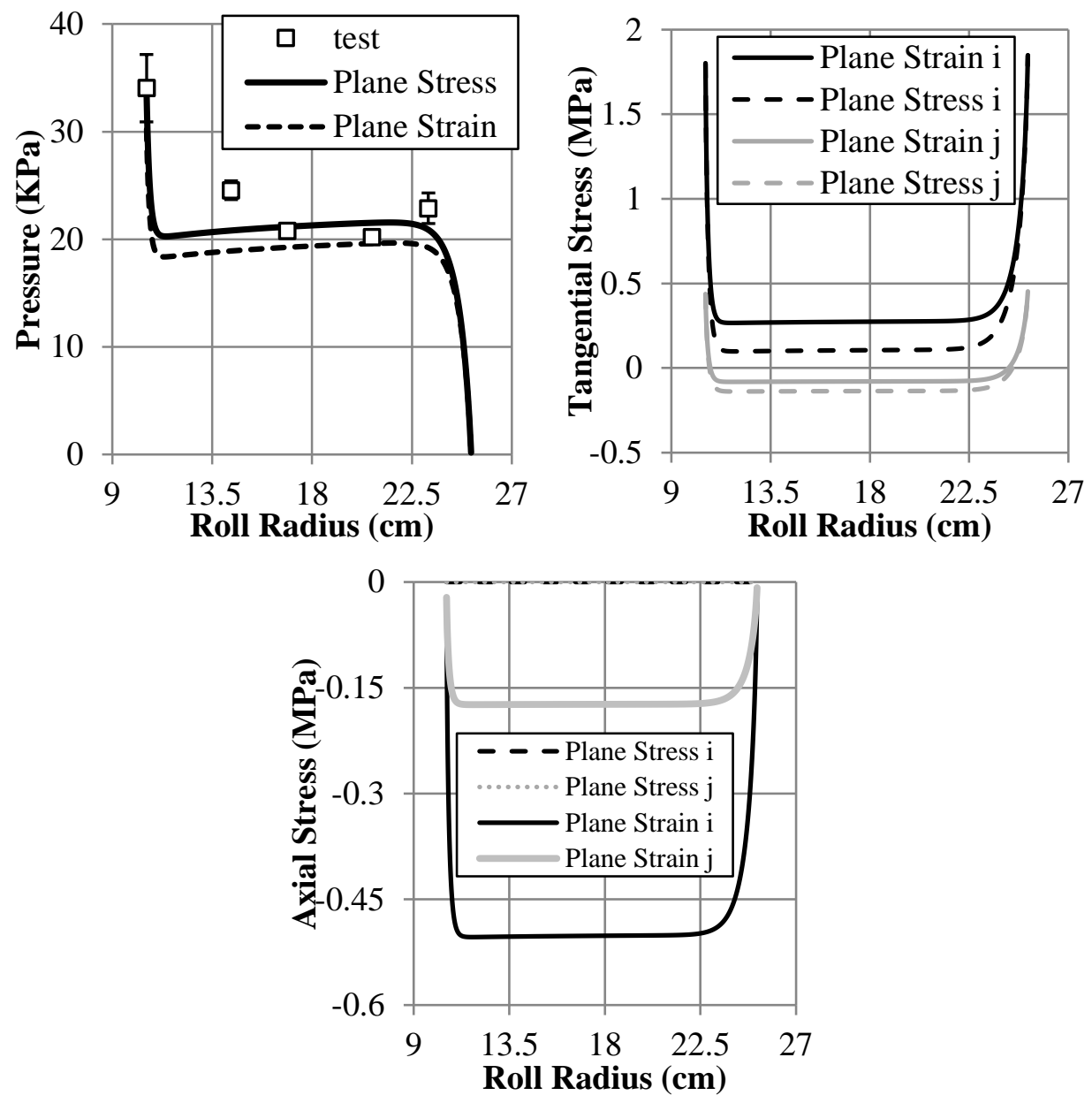

Figure 6 - Model and Test Results: Non-Strain Matched Case A 

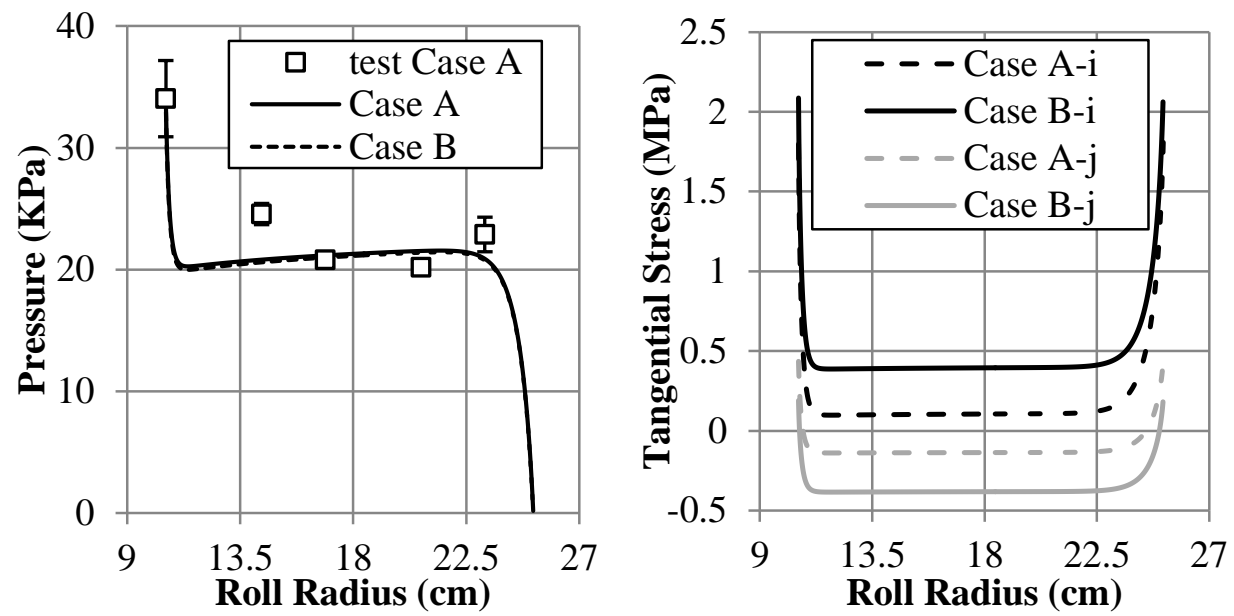

Figure 7 - Effects of Lamination Tension, Non-Strain Matched Cases.

\section{CONCLUSIONS}

A 1D finite element model that can allow either plane stress or plane strain material behaviors was developed and verified against test data acquired for winding newsprint. The $1 \mathrm{D}$ finite element model was extended to accommodate laminate webs. It was found to be necessary to employ a condensation method to allow this model to function correctly. The model was validated for cases where the web strains were matched at the laminator and for cases where the strains were intentionally not matched. Even though the model is capable of modeling plane stress or plane strain behaviors it is incapable of predicting which of these behaviors exist in a given wound roll. This could be predicted with 2D winding models that are extended to winding laminates. Results presented herein show that the negative axial stresses associated with plane strain are substantial and should be accounted for when axial buckling defects are witnessed. For laminate webs it has been shown that the lamination tensions and the winding tension can affect the tangential stresses in the wound roll which will have impact on creep and curl defects.

\section{REFERENCES}

1. Hakiel, Z., “Nonlinear Model for Wound Roll Stresses,” TAPPI Journal, Vol. 70, 1987, pp. 113-117.

2. Willett, M. S., and Poesch, W. L., "Determining the Stress Distributions in Wound Reels of Magnetic Tape using Nonlinear Finite Difference Approach,” Journal of Applied Mechanics, ASME, Vol. 55, 1988, pp. 365-371.

3. Pfeiffer, J. D., “Internal Pressures in a Wound Roll of Paper,” TAPPI Journal, Vol. 48, No. 8, 1966, pp. 342-347.

4. Mollamahmutoglu, C., and Good, J. K., "Analysis of Large Deformation Wound Roll Models,” ASME Journal of Applied Mechanics, Vol. 80, 2013, pp. 041016-111. 
5. Pfeiffer, J. D., “Measurement of the K2 Factor for Paper,” Tappi Journal, Vol. 64, No. 4, 1981, pp. 105-106.

6. Good, J. K., Pfeiffer, J. D., and Giachetto, R. M., "Losses in Wound-On-Tension in the Center Winding of Wound Rolls," ASME Applied Mechanics Division, Vol. 149, Web Handling 1992, 1992, pp. 1-12.

7. Cole, K. A., and Hakiel, Z., "A Nonlinear Wound Roll Model Accounting for Widthwise Web Thickness Nonuniformities," Proceedings of the Web Handling Symposium, ASME Applied Mechanics Division. Vol. 149, 1992, pp. 13-24.

8. Kedl, D. M., "Using a Two Dimensional Winding Model to Predict Wound Roll Stresses that Occur due to Circumferential Steps in Core Diameter or to Cross Web Caliper Variation," Proceedings of the First International Conference on Web Handling, Web Handling Research Center, Oklahoma State University, 1991, pp. 99-112.

9. Lee, Y. M., and Wickert, J. A., "Stress Field in Finite Width Axisymmetric Wound Rolls,” ASME Journal of Applied Mechanics, Vol. 69, No. 2, 2002, pp. 130-138.

10. Arola, K., and von Hertzen, R., "Two Dimensional Axisymmetric Winding Model for Finite Deformation,” Computational Mechanics, Vol. 40, No. 6, 2007, pp 933947.

11. Hoffecker, P., and Good, J. K., "Tension Allocation in a Three Dimensional Wound Roll,” Proceedings of the Eighth International Conference on Web Handling, Web Handling Research Center, Oklahoma State University, Stillwater, Oklahoma, 2005, pp. 565-581.

12. Mollamahmutoglu, C., and Good, J. K., "Modeling the Influence of Web Thickness and Length Imperfections Resulting from Manufacturing Processes on Wound Roll Stresses," CIRP Journal of Manufacturing Science and Technology, Vol. 8, January 2015, pp. 22-33.

13. Good, J. K., Mollamahmutoglu, C., Markum, R. and Gale, J. W., "Residual Winding Stresses Due to Spatial Web Thickness Variation,” ASME Journal of Manufacturing Science and Engineering, Vol. 139, No. 3, March 2017.

14. Cook, R. D., Malkus, D. S., and Plesha, M. E., Concepts and Applications of Finite Element Analysis, $3^{\text {rd }}$ edition, John Wiley and Sons, 1989, pp.228-229. 